
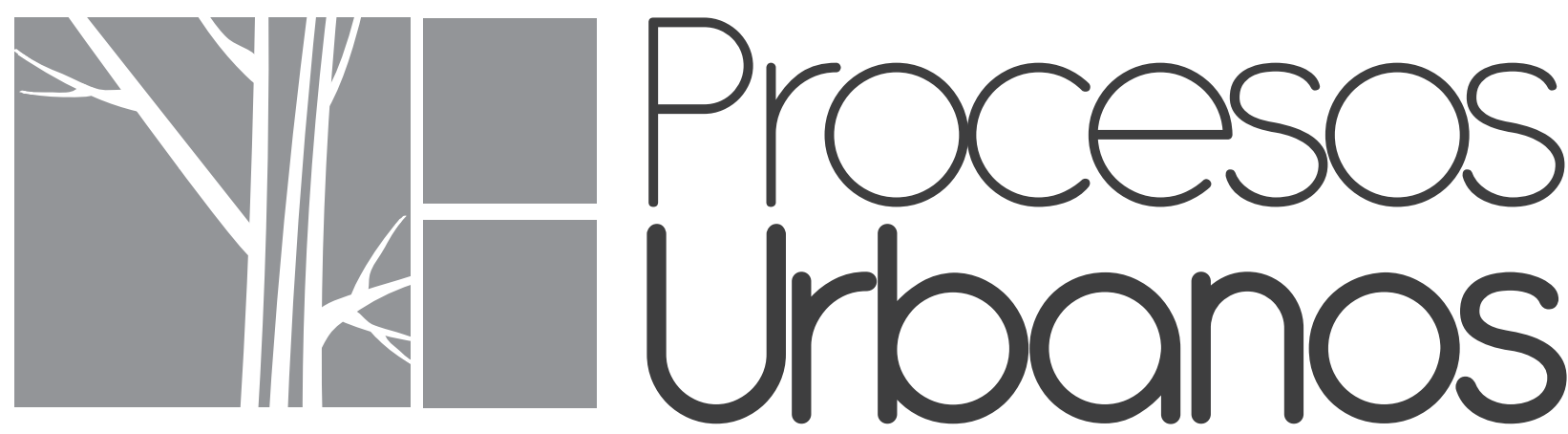

Revista de Divulgación Científica 


\section{Revista Procesos Urbanos}

Facultad de Ciencias Básicas, Ingeniería y Arquitectura.

ISSN: 2422-085X

Sincelejo, Enero - Diciembre de 2014

Piedad Martínez Carazo

Rectora

\section{Lidia Flórez de Albis}

Vicerrectora Académica

\section{Jhon Víctor Vidal}

Director de Investigaciones

\section{Pedro Arturo Martínez Osorio}

Editor

\section{Comité Editorial:}

PhD. Alexander Niño Soto. Universidad Antonio Nariño

PhD. Jorge Ramírez Nieto. Universidad Nacional de Colombia

Msc. Juan Carlos Pergolis. Universidad Católica de Colombia

PhD. Piedad Martínez Carazo. Corporación Universitaria del Caribe, CECAR

PhD. Emiro F. Martínez. Universidad de York, Canadá

\section{Comité Científico:}

Msc. Gloria Aponte García. UPB, Medellín

Msc. Jorge Vásquez Muñoz. UPB, Medellín

PhD. Jorge Gómez Ricardo, Universidad del Magdalena

\section{Editorial CECAR}

\section{Libia Narváez Barbosa}

Coordinadora Editorial

\section{Eduardo Támara Galván}

Corrector de Estilo

\section{Alejandrina Jaramillo y Roger Goez Castillo}

Diseño Gráfico

Diagramación e Impresión:GRÁFICAS DEL CARIBE S.A.S.

Cra. 1B No. 40-42 Montería Tel. (57) (4) 7826622 Telefax (57) (4) 7817112

Email: diseno@graficaribe.co

\section{Dirección:}

Carretera Troncal de Occidente

Kilómetro 1 Vía a Corozal

Tel: 280402928040172804018 Ext 1115

www.cecar.edu.co

http://cecar.edu.co/revista-procesos-urbanos 


\title{
TRIBUTACIÓN A LA PROPIEDAD RAÍZ: INGENTE CARGA FINANCIERA QUE ATENTA CONTRA EL FOMENTO DE LA ACTIVIDAD INMOBILIARIA
}

\author{
Taxing to the real estate: Tremendous financial burden on that threatens the promotion \\ of housing activity
}

Jorge Augusto Gómez Ricardo ${ }^{1}$

Fecha: Recibido enero 20 de 2014 / Aceptado junio 3 de 2014

\section{RESUMEN}

Se parte de la hipótesis en la que el sistema tributario colombiano se constituye en un esquema ampliamente oneroso que afecta directamente a los intereses de los oferentes y demandantes de propiedad raíz en Colombia. Aspecto que redunda de manera nociva sobre designios de equidad, eficiencia, crecimiento y estabilidad para la sociedad. Obstaculiza el avance de las municipalidades y de la actividad económica en general, en especial afecta al sector de la construcción, y por ende el desarrollo de las ciudades. El método desarrollado recurre al esquema de investigación basado en observaciones sobre el entorno real, acompañado de sus bases jurídicas, experiencias e interpretaciones hilvanadas por tratadistas de la disciplina fiscal. Se plantean hipótesis, se recurre a estrategias para descubrir propiedades y características del tema objeto de estudio y se concluye acorde con la hipótesis. Como conclusión se tiene que el exceso de gravámenes incide de manera negativa sobre políticas públicas orientadas hacia la distribución equitativa de ingresos y riquezas, crecimiento económico, estabilidad de precios, empleo y asignación eficiente de los recursos disponibles. En particular, el sector de la construcción es el más afectado con la cascada de tributos que se generan en Colombia, incidiendo sobre la oferta y la demanda, afectando tanto a usuarios, adquirientes y empresarios del ramo. Se sugiere reducir las cargas impositivas para asegurar mayor progreso social y fomento de la actividad edificadora y para asegurar efectivo recaudo del impuesto predial.

Palabras Clave: Tributo, Estado, finanzas, propiedad raíz, equidad, eficiencia, estabilidad, crecimiento, cascada tributaria.

${ }^{1}$ Doctor en Derecho (Ph. D.), U. Externado de Colombia. Master en Economía e Instituciones, Universidad de Roma II, Tor Vergata. Master en Estudios Políticos, Universidad Javeriana. Economísta, Universidad Externado de Colombia. Abogado, Universidad La Gran Colombia. Correo electrónico: jgomezricardo@yahoo.com Docente: CECAR; U. Magdalena, U. Externado de Colombia, U. Javeriana, U. Nacional, U. Roma II, U. Táchira, entre otras. 


\section{ABSTRACT}

It begins of the hypothesis in which the Colombia's tributary system is constituted in a widely onerous scheme that affects directly to the interests of the offers and plaintiffs of real estate in Colombia. Aspect that results in a harmful way on plans of equity, efficiency, growth and stability for the society. It blocks the advance of the municipalities and of the economic activity in general, especially sympathetic on the sector of the construction, and for the development of the cities. The developed method goes to the scheme of investigation based on observations on the real environment accompanied of his juridical bases, experiences and interpretations tacked by commentators of the fiscal discipline. Hypotheses appear, one resorts to strategies to discover properties and characteristics of the topic or object of study and it concludes according to hypothesis. As a conclusion has that the excess of charges affects in a negative way on public policies orientated towards the equitable distribution of income and wealths, economic growth, price stability, employment and efficient assignment of the available resources. Especially, the sector of the construction is most affected by the sequel of taxes that are generated in Colombia, affecting on the offer and the demand, affecting so much users, acquirers and businessmen of the branch. It is suggested to reduce the tax burdens to assure major social progress and promotion of the edifying activity and to assure cash collection of the predial tax.

Keywords: Tax, State, finance, real estate, equity, efficiency, stability, growth, tributary sequel.

\section{INTRODUCCIÓN}

En el devenir histórico han existido múltiples tributos que afectan a la propiedad raíz en todas las esferas estatales (nacional, departamental, municipal y distrital), que han ocasionado efectos nocivos a la sociedad colombiana. Han perjudicado a los beneficiarios de los impuestos: Pérdida de autonomía, deterioro en su capacidad de recaudo y proclividad a la evasión y la elusión. También, han originado secuelas negativas sobre los sujetos pasivos de los tributos al originar esquema oneroso, con efectos indeseables, injusticia, ineficiencia, inestabilidad y desestímulo a la inversión productiva.

En el marco institucional colombiano se han impulsado avances significativos. Se resalta, en primer lugar, la decisión de fusionar varios gravámenes, lo que permitió el advenimiento del impuesto predial unificado, $y$, en segundo término, la disposición de la Asamblea Constituyente reunida en 1991 de otorgar a los municipios la propiedad exclusiva de los gravámenes que afectan a la propiedad raíz, hechos que deben ser analizados con sensatez y prudencia, dado que pueden catalogarse como evolución; sin embargo, en la práctica todavía persisten cascadas de tributos que se convierten en talanquera para el desarrollo de la actividad edificadora.

\section{METODOLOGÍA}

Se recurre a esquema de investigación basado en observaciones sobre el entorno real, acompañado de sus bases jurídicas, experiencias e interpretaciones hilvanadas por tratadistas de la disciplina fiscal. Es posible circunscribirlo en el método científico, puesto que se plantea hipótesis, se recurre a estrategia para descubrir propiedades y características del tema objeto de estudio y se concluye acorde con la hipótesis. Corresponde a técnica cualitativa, al proponer relación entre el objeto y el sujeto de estudio.

\section{RESULTADOS}

Históricamente ha existido preocupación por la cascada de tributos que afectan a la propiedad raíz, que supone efectos onerosos e incidencias especulativas, que alcanzan dimensiones preocupantes dada la existencia de contexto de disponibilidad ilimitada de inmuebles. Esta preocupación 
ha sido pródigamente debatida, al punto que diferentes políticas públicas han impulsado tendencias a fusionar gravámenes.

En efecto, con el propósito de evitar la cascada de tributos, la Ley 44 de 1990 tuvo a bien fusionar cuatro gravámenes existentes en aquella época: los impuestos predial, a parques y arborización, estratificación socioeconómica y la sobretasa de levantamiento catastral, que propició el surgimiento del impuesto predial unificado. Decisión pública entendida como alivio frente al exceso de cargas tributarias.

Sin embargo, en la realidad persisten aún numerosos gravámenes que, afectan a la propiedad raíz. Se destacan sólo en el ámbito municipal: Contribuciones de valorización, especial de obras públicas, especial de obras públicas para seguridad y convivencia y desarrollo municipal, impuestos a delineación urbana y alumbrado público, sobretasas para corporaciones autónomas regionales y para áreas metropolitanas y tasas de servicios públicos. Además, las entidades territoriales recaudan recursos por emisión de estampillas, se resaltan las destinadas a: salud, cultura, electrificación rural, universidades, adulto mayor, entre otras, que si bien no afectan directamente a la propiedad raíz, tienen incidencia sobre los contratos estatales relativos a venta o arriendo de inmuebles o a ejecución de obras civiles.

También, existen otros tributos de índole nacional y departamental que afectan de manera indirecta a los inmuebles. En el entorno departamental se resaltan: Impuestos de registros y anotación, sobretasa al impuesto de registro $y$ anotación y contribuciones departamentales de valorización y especial de obras públicas para seguridad y convivencia. En el contexto nacional, se aplican variados gravámenes que afectan la propiedad raíz, que no consultan a la propiedad como índice de capacidad de pago: impuesto básico a la renta y complementario a las ganancias ocasionales y, de forma eventual, se ha aplicado el impuesto al patrimonio, impuestos de timbre y contribución de valorización del orden nacional.
La Constitución Política de Colombia dispuso en su artículo 317: "Sólo los municipios podrán gravar la propiedad inmueble...". Así, se promovió la autonomía fiscal y el mejoramiento de la capacidad financiera de los municipios. Sin embargo, esta disposición se orienta sólo a la aplicación de los tributos cuya capacidad de pago es la propiedad. En cambio, no incide sobre los gravámenes que examinan otros indicadores de la capacidad económica: ingresos, riqueza y consumo. Así, en el ámbito departamental y nacional persiste gran cúmulo de tributos que afectan a los predios.

Los efectos de esta amplia cascada tributaria son propiciar excesiva especulación sobre los inmuebles, aunada a las secuelas derivadas de la oferta inelástica por la tierra. Es claro que la población aumenta en gran proporción y, en consecuencia, el suelo es cada vez menos accesible para los diferentes usos económicos. Así, los efectos especulativos conciernen al crecimiento exagerado de los precios de los predios tranzados en el mercado inmobiliario, muy superior a los registrados por otros bienes.

Este panorama justifica reducir la maraña tributaria que afecta a la propiedad raíz. Existe incertidumbre en observancia de los objetivos de equidad, estabilidad, eficiencia y crecimiento. Se debe pensar más bien en políticas públicas orientadas a estimular la actividad edificadora, mediante incentivos tributarios y subsidios sociales, con énfasis en la vivienda popular y la pequeña propiedad agrícola.

Los efectos sobre la equidad se pueden resumir en los siguientes aspectos: dificultad cada vez mayor, para las familias de bajos recursos de disponer de vivienda o tierra cultivable. Además, la actividad de la construcción y de obras civiles promueven empleos productivos, que dadas estas talanqueras frenan la opción de mejorar los índices de ocupación. Estos factores repercuten en mayor brecha social, generando condiciones de inconformidad y discrepancia y propiciando entorno de inestabilidad económica y social. 
Trasgrede la estabilidad, porque al negar la opción de proyectos de infraestructura se incide nocivamente sobre los grados de ocupación. La infraestructura es fuente esencial para generar empleos.

También, frena la opción de asegurar el crecimiento económico, al desestimular la posibilidad de propiciar programas y proyectos esenciales, fundamentales para promover el desarrollo urbanístico.

Redunda de forma nociva sobre la eficiencia, porque el exceso de costos trasgrede los resultados financieros de los inversionistas, incidiendo negativamente en las condiciones optimas de producción y consumo, afecta el denominado óptimo de Pareto (1946).

El impuesto predial unificado es la principal fuente de financiación de las municipalidades y se justifica como único gravamen a los predios. Su hecho generador se relaciona con el derecho de propiedad (pertenencia o posesión). Son beneficiarios (sujetos activos) del tributo: Municipios y distritos; debe destinarse el $10 \%$ del recaudo a subsidio para servicios de acueducto, alcantarillado y aseo (artículo 7 de la Ley 44 de 1990 y artículo 100 de la Ley 142 de 1994). El sujeto pasivo es el propietario o poseedor del inmueble. El objeto principal de la obligación es pagar suma determinada de dinero, obligación de dar. Existen además obligaciones secundarias, en su mayoría de hacer, como declarar; también existen algunas obligaciones de no hacer, como no incurrir en extemporaneidad en el pago del tributo para evitar multas. Se causa a partir del 1 de enero de cada anualidad y existen fechas límites de pago fijadas por la administración; el pago extemporáneo suscita multas (mora); algunos municipios establecen beneficios por pronto pago. Su base gravable es el auto-avalúo, reglado en la declaración anual; se estipuló que la base mínima es el avalúo catastral, fijado por el Instituto Geográfico Agustín Codazzi y las oficinas de catastro de Bogotá, Medellín, Cali y Antioquia. El Ministerio de Hacienda reajusta cada año el avalúo catastral con base en la inflación. Las tarifas son establecidas por los concejos municipales y distritales mediante proyecto de acuerdo presentado por el ejecutivo, conforme al rango: 0,1 a 1,6\%, acorde con estratos socioeconómicos, usos del suelo y antigüedad de formación y actualización del catastro. Las tarifas podrían ser superiores para predios urbanizados no edificados y urbanizables no urbanizados, sin ser superior el 3,3\%. Se aplican tarifas mínimas a la vivienda de interés social y a la pequeña propiedad rural. Se originan nuevos avalúos por construcción, formación de predios y cambio de destino económico.

Se resaltan algunas deficiencias en la aplicación de este tributo en relación con la equidad:

a. El impuesto predial unificado para efectos de determinar su base gravable considera el valor bruto del activo, ignorando pasivos que afectan dicha propiedad. Se violan los principios de equidad expresados por Simons (1936), en el sentido que las personas que adquieren su propiedad sin hipoteca pagan el mismo impuesto predial que otra que la consigue con deuda, y que además está sujeta a la onerosa carga de la deuda hipotecaria. Se presenta interesante contra-argumento, dada la incidencia de la inflación, que de forma histórica ha sido representativa en América Latina. Así, la valorización del predio resulta ventajosa a las propiedades con deudas hipotecarias. El porcentaje de ganancia es más alto para el propietario que adquiere el inmueble a crédito, que para aquél que lo adquiere sin deuda: "La inflación genera utilidades extraordinarias a favor de los propietarios de la tierra" (Ministerio de Hacienda y Crédito Público, 1972), lo que proporciona mayor beneficio a propietarios con obligaciones hipotecarias. Se considera que quien reciba beneficio especial contribuya a sufragar las necesidades comunes.

Para ilustrar este aspecto, se recurre a la próxima ilustración: Supóngase dos individuos que adquieren propiedades idénticas en proyecto de casas en serie. Las características básicas de este caso 
hipotético son: Precio de mercado de casa $=$ $\$ 200.000 .000$, valoración anual por efectos inflacionarios $=10 \%(\$ 20.000 .000) . \mathrm{EI}$ primer individuo adquiere su casa sin hipoteca, por ello el beneficio recibido al año de su compra es del $10 \%(\$ 20.000 .000$ / \$200.000.000). El segundo personaje adquiere su casa con hipoteca del $70 \%$ $(\$ 140.000 .000)$, abonó con recursos propios sólo el 30\% (\$60.000.000), siendo su margen de ganancia del 33,3\% $(\$ 20.000 .000$ / $\$ 60.000 .000)$. En este ejercicio sólo se tuvo en cuenta los beneficios derivados de la valorización producto de la inflación, éstos podrían ser superiores si se tuviese en cuenta la especulación, factor difícil de cuantificar, pero de gran relevancia en el mercado inmobiliario por efectos de la presencia de oferta limitada del suelo.

b. En la vida práctica, los principios de equidad también se violan al otorgarle prelación al avalúo catastral, al ser considerado por la norma como base mínima en la determinación del impuesto predial; la mayoría de propietarios declaran el gravamen sobre dicha base, que suele ser inapropiada frente a la realidad económica, dado que presenta deficiencias en su actualización $y$ formación, que significa que suele ser lento para captar cambios originados por el mercado o modificaciones ejercidas por el propio propietario $y$, además, resulta ser caprichoso. Dos personas que tienen propiedades con el mismo valor económico, tributan de manera diferente, porque se les tasa según esos avalúos o en algunos casos propiedades más costosas pagan gravámenes menos onerosos que otras menos valiosas. Tratadistas señalaron deficiencias del avalúo catastral, al revelar problemas de gestión en la actualización y formación por parte de la autoridad catastral: Vásquez (1971), Hey (1974), Lewis (1979), Netzer (1966).

Al promulgarse la Ley 14 de 1983 el Ejecutivo se propuso corregir dichas deficiencias, al establecer cuatro mecanismos que permitieran mantener actualizados los avalúos: Auto-avalúo, avalúo automático retroactivo, reajuste automático hacia el futuro y avalúo administrativo. Asimismo, mediante Ley 44 de 1990 el legislador adoptó la declaración de impuesto predial unificado o auto-avalúo. Así, se recurrió al propio contribuyente para solucionar las deficiencias de la autoridad estatal.

La tendencia a esquemas de eficiencia fiscal, autogeneración de recursos, propulsados frente a la crisis financiera de entidades territoriales vivida en Colombia en las últimas décadas del siglo XX, impulsó acciones de gobiernos municipales tendientes a generar avalúos excesivos, que superan en ocasiones a los precios comerciales. Se han originado situaciones en las que las personas tributan sobre avalúos altos y al vender sus inmuebles reciben recursos bastante inferiores a los avalúos oficiales. Situación que colige pago de impuestos superiores a los que corresponden.

No obstante, algunos autores justifican el exceso de tributación a la propiedad raíz. Al respecto, Llorente (1972), Ministro de Hacienda, al presentar su propuesta de reforma fiscal de 1972, formuló que la propiedad raíz absorbe esfuerzos de la comunidad. En gran proporción se valoriza el predio por esos esfuerzos y, por tanto, es justo que quienes tienen inmuebles devuelvan a la comunidad los beneficios recibidos, mediante el impuesto predial. Otros tratadistas justifican el gravamen, al considerar que en Colombia la propiedad raíz en el devenir histórico ha sido factor característico de la concentración de la riqueza. Al respecto, Hey (1974) esbozó: "La propiedad raíz es índice aproximado de la capacidad de pagar impuestos y su existencia es muy difícil de ocultar a la administración de impuestos. Gran parte de las tierras están concentradas en manos de ricos". En el mismo sentido, Urrutia (1974), al referirse en específico a la propiedad rural, observó: "No cabe duda de que la mala distribución de la propiedad rural es causa principal de la gran dispersión de los ingresos agrícolas. Los ingresos altos corresponden a personas con fincas grandes, y los grupos más pobres son jornaleros sin tierras y las 30.000 personas que tienen fincas de menos de una hectárea". 
PROCESOS URBANOS - Revista de Divulgación Científica Vol. 1 Enero - Diciembre 2014 (103 - 112)

c. En el ámbito histórico han existido variados tributos que afectan a la propiedad raíz en todas las esferas (nacional, departamental y municipal o distrital), ocasionando efectos nocivos a los sujetos activos o beneficiarios del impuesto predial, como son: pérdida de autonomía, deterioro en su capacidad de recaudo y proclividad a evasión y elusión. Se debe fortalecer el predial y eliminar los otros.

d. Con la finalidad de asegurar progresividad, los municipios cuentan con importante instrumento: Aplicar tarifas diferenciales, herramienta poco utilizada por gestores públicos de entidades territoriales, justificando complejidad de índole administrativa. Las tarifas diferenciales tienen como finalidad asegurar equidad (gravar más a la propiedad lujosa), castigar predios no utilizados con fines económicos, penar a predios que coadyuven a efectos nocivos (contaminación, congestión, ruido, inmoralidad, desorden, etc.) y afectar fuertemente a lotes de engorde, que frenan el desarrollo municipal.

El artículo 3 de la Ley 44 de 1990, dispuso: "La tarifa del impuesto predial unificado será fijada por los concejos y oscilará entre 1 y 16 por mil del respectivo avalúo... Las tarifas en cada municipio deberán establecerse de manera diferencial y progresiva, teniendo en cuenta: a) estratos socio-económicos; b) usos del suelo en el sector urbano; c) antigüedad en la formación y actualización del catastro... A la vivienda popular y a la pequeña propiedad rural destinada a producción agropecuaria se le aplicarán las tarifas mínimas establecidas por el respectivo concejo... Las tarifas a predios urbanizables no urbanizados y urbanizados no edificados podrán ser superiores al límite señalado, sin exceder de 33 por mil".

El artículo 67 de la Resolución 2555 de 1988 estableció clasificación catastral de los predios por su destinación económica.

Ilustración de estructura de tarifas diferenciales en la aplicación del impuesto predial unificado es la experiencia de
Bogotá (Acuerdo 039 de 1993, artículo 4), que estableció las siguientes tarifas:

El Acuerdo 105 de 2003, emitido por el concejo del Distrito Capital, mantuvo las características de tarifas diferenciales para el impuesto predial en Bogotá, adecuándolas al plan de ordenamiento territorial. En su artículo 1 diferenció dos categorías de predios: 1) Residenciales urbanos y rurales y 2) no residenciales. El Consejo de Estado (2010) decretó la nulidad parcial de esta norma. Con base en esta clasificación instauró la estructura de tarifarias diferenciales, en los siguientes términos:

El artículo 23 de la Ley 1450 de 2011 modificó al artículo 4 de la Ley 44 de 1993, al establecer que las tarifas diferenciales del impuesto predial unificado deben consultar las Unidades de Valores Tributarios (UVT), fijadas por el Ministerio de Hacienda y Crédito Público. La tarifa se obtiene acorde con la siguiente fórmula: Base gravable (avalúo catastral) por tarifa impositiva menos ajuste por progresividad (corresponde al ajuste tarifario establecido en el artículo 2 del Acuerdo 105 de 2003).

La progresividad del impuesto predial para personas naturales es destacada por Linn (1979), que basado en estudios empíricos concluyó que el peso del tributo se incrementa al aumentar el ingreso. Esta consideración le permite aseverar que la elasticidad del recaudo del gravamen con respecto del ingreso es mayor que uno, en especial en los grandes centros urbanos. Contrario sensu, demostró que el impuesto predial aplicado a empresas es regresivo, al afectar menos a los grupos sociales de alto nivel de ingresos.

De otro lado, aspecto de crucial importancia es la consideración de la relación entre mayor productividad y mejor distribución del ingreso. Se considera que si los procesos económicos ejercidos en el suelo son eficientes, conducen a mayor avance económico, que implica mayor producción,

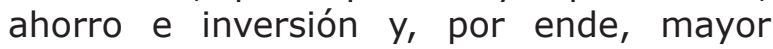
empleo e ingreso, con lo que se favorecen las condiciones de la población. 
Tabla 1. Estructura de tarifas diferenciales en la aplicación del impuesto predial unificado. Experiencia de Bogotá. (Fuente Acuerdo 039 de 1993, artículo 4)

\begin{tabular}{|c|c|}
\hline TIPOS DE PREDIOS & TARIFA POR MIL \\
\hline \multicolumn{2}{|c|}{ PREDIOS RURALES } \\
\hline Predios rurales & 5,0 \\
\hline Predios suburbanos & 20,0 \\
\hline Predios rurales institucionales & 5,0 \\
\hline \multicolumn{2}{|c|}{ PREDIOS URBANOS } \\
\hline \multicolumn{2}{|c|}{ Predios edificados residenciales, estratos 1,2 y 3} \\
\hline Hasta 70 m cuadrados de construcción & 4,0 \\
\hline Más de 70 m cuadrados de construcción & 5,0 \\
\hline \multicolumn{2}{|l|}{ Predios edificados residenciales, estrato 4} \\
\hline Hasta 100 m cuadrados de construcción & 6,0 \\
\hline Más de 100 m cuadrados de construcción & 7,0 \\
\hline \multicolumn{2}{|l|}{ Predios edificados residenciales, estrato 5 y 6} \\
\hline Hasta 220 m cuadrados de construcción & 7,0 \\
\hline De 220 y hasta 300 m cuadrados de construcción & 8,0 \\
\hline Más de $300 \mathrm{~m}$ cuadrados de construcción & 8,5 \\
\hline \multicolumn{2}{|c|}{$\begin{array}{ll}\text { INDUSTRIALES } \\
\end{array}$} \\
\hline Alto impacto & 10,0 \\
\hline Medio impacto & 9,0 \\
\hline Bajo impacto & 8.5 \\
\hline \multicolumn{2}{|c|}{ COMERCIALES } \\
\hline Local & 8,0 \\
\hline Zonal & 8,5 \\
\hline Metropolitano & 9,5 \\
\hline \multicolumn{2}{|c|}{ CIVICO INSTITUCIONALES } \\
\hline Local & 5,0 \\
\hline Zonal & 6,0 \\
\hline Metropolitano & 6,5 \\
\hline \multicolumn{2}{|c|}{ URBANIZABLES NO URBANIZADOS Y URBANIZABLES NO EDIFICADOS } \\
\hline Hasta 100 metros cuadrados & 12,0 \\
\hline De 100 y hasta 240 metros cuadrados & 14,0 \\
\hline De 240 y hasta 1.000 metros cuadrados & 16,0 \\
\hline De 1.000 y hasta 6.000 metros cuadrados & 18,0 \\
\hline Más de 6.000 metros cuadrados & 20,0 \\
\hline \multicolumn{2}{|c|}{ OTROS USOS } \\
\hline Entidades financieras & 15,0 \\
\hline Empresas industriales y comerciales del Estado & 8,5 \\
\hline
\end{tabular}


PROCESOS URBANOS - Revista de Divulgación Científica Vol. 1 Enero - Diciembre 2014 (103 - 112 )

Tabla 2. Predios residenciales urbanos y rurales. (Elaboración del autor)

\begin{tabular}{|c|c|}
\hline \multicolumn{2}{|c|}{ PREDIOS RESIDENCIALES URBANOS Y RURALES } \\
\hline \multicolumn{2}{|c|}{ RESIDENCIALES URBANOS ESTRATOS 1, 2 y 3} \\
\hline Estratos 1 y 2 con avalúo catastral entre $\$ 6.400 .000$ y $\$ 42.500 .000$ & $2 \%$. \\
\hline Base gravable inferior o igual a $\$ 22.500 .000$ & $4 \%$ \\
\hline Base gravable superior a $\$ 22.500 .000$ & $6 \%$ \\
\hline \multicolumn{2}{|l|}{ RESIDENCIALES URBANOS ESTRATO 4} \\
\hline Base gravable inferior o igual a $\$ 53.700 .000$ & $6 \%{ }_{0}$. \\
\hline Base gravable superior a $\$ 53.700 .000$ & $7,5 \%$ \\
\hline \multicolumn{2}{|l|}{ RESIDENCIALES URBANOS ESTRATOS 5 y 6} \\
\hline Base gravable inferior o igual a $\$ 142.400 .000$ & $7 \%$ \\
\hline Base gravable superior a $\$ 142.400 .000$ & $9,5 \%$ \\
\hline \multicolumn{2}{|l|}{ RESIDENCIALES RURALES } \\
\hline Base gravable inferior o igual a $\$ \mathbf{1 8 . 0 0 0 . 0 0 0}$ & $4 \%$ \\
\hline Base gravable superior a $\$ 18.000 .000$ & $7 \%$ \\
\hline \multicolumn{2}{|l|}{ PREDIOS NO RESIDENCIALES } \\
\hline \multicolumn{2}{|l|}{ COMERCIALES EN SUELO RURAL O URBANO } \\
\hline Base gravable inferior o igual a $\$ 50.000 .000$ & $8 \%{ }_{0}$ \\
\hline Base gravable superior a $\$ 50.000 .000$ & $9,5 \%$ \\
\hline Financieros & $15 \%$ \\
\hline \multicolumn{2}{|l|}{ INDUSTRIALES EN SUELO URBANO O RURAL } \\
\hline Industriales bajo impacto & $8,5 \%$ \\
\hline Industriales medio impacto & $9 \%$ \\
\hline Industriales & $10 \%$ \\
\hline \multicolumn{2}{|l|}{ PREDIOS ROTACIONALES } \\
\hline Propiedad particular & $6 \%$ \\
\hline Propiedad de entes públicos & $5 \%$ \\
\hline \multicolumn{2}{|c|}{ PREDIOS URBANIZABLES NO URBANIZADOS Y URBANIZADOS NO EDIFICADOS } \\
\hline Base gravable inferior o igual a $\$ 15.000 .000$ & $12 \%$ \\
\hline Base gravable superior a $\$ 15.000 .000$ & $33 \%$. \\
\hline \multicolumn{2}{|l|}{ PREDIOS NO URBANIZABLES } \\
\hline No urbanizables & $4 \%$ \\
\hline \multicolumn{2}{|c|}{ PEQUEÑA PROPIEDAD RURAL DESTINADA A PRODUCCIÓN AGROPECUARIA } \\
\hline Pequeña propiedad rural destinada a la producción agropecuaria & $4 \%$ \\
\hline \multicolumn{2}{|l|}{ PREDIOS RURALES } \\
\hline Predios rurales & $10 \%$. \\
\hline
\end{tabular}

Algunos autores, incluidos Hirshman (1968) y Currie (1974), plantearon que los tributos que afectan a la propiedad raíz son buenos instrumentos para promover el mejor uso de las tierras e incrementar su productividad. Consideran que para cubrir los costos del tributo es preciso asegurar eficiencia en el proceso productivo que se ejerce en cada inmueble.

También la OEA y el BID (1973) recomendaron el uso de impuestos sobre la tierra para asegurar mayor eficiencia en la actividad agropecuaria. Propuso esquema de tributo basado en la renta potencial. Planteó: "La ventaja de gravar la renta potencial, en lugar de la renta real, radica en estímulo mayor, por el efecto ingreso sin efecto sustitución. En otras palabras, su efecto sobre la productividad no podría asegurarse si se grava la renta real, en tal caso el efecto sustitución podría contrarrestar en medida apreciable el efecto ingreso. Al gravarse la renta potencial, por cada unidad adicional producida no se 
aumenta el monto del impuesto. De otro lado, la imposición de la renta potencial de la tierra tiene la peculiaridad de no permitir evasión mediante reducción de la base del impuesto. La tierra es factor limitado, lo que no ocurre con el capital, cuya acumulación puede ser desestimulada si se grava su renta potencial. De ahí, que sea aconsejable acudir al impuesto sobre la renta potencial de la tierra, sin incluir al capital agrícola".

La Ley 1430 de 2010, artículo 60, instauró el carácter real del impuesto predial unificado. Estableció que recae sobre bienes raíces y se hace efectivo independiente de su propietario. Dispuso que para otorgase escritura pública en actos de traspaso de inmuebles, deberá certificarse ante notario el pago del impuesto predial.

Los propósitos del impuesto predial son variados según Low y Gómez (1986), se resaltan: 1) Generar importantes fuentes de financiación a los municipios, afín con Froomkin (1968), Hey (1974), Netzer(1966), Lewis (1979). 2) Impulsar mayor actividad económica en el sector rural, conforme con Hirshman (1968), Currie (1974), BID (1952). 3) Propulsar mejor utilización del suelo en áreas urbanas, acorde con Hicks (1968), Lewis (1979), Hey (1974).

\section{CONCLUSIONES}

En efecto, el exceso de gravámenes que afectan la propiedad raíz es nocivo a importantes propósitos de la política económica y social, incidiendo de manera negativa sobre políticas públicas orientadas hacia la distribución equitativa de ingresos y riquezas, crecimiento económico, estabilidad de precios y empleo y asignación eficiente de los recursos disponibles. En particular, el sector de la construcción es el más afectado con la cascada de tributos que se generan en Colombia, incidiendo sobre la oferta y la demanda, afectando tanto a usuarios, adquirientes y empresarios del ramo. Se sugiere reducir las cargas impositivas para asegurar mayor progreso social y fomento de la actividad edificadora y para asegurar efectivo recaudo del impuesto predial. Se debe actuar de forma inversa, más bien estimular la actividad constructora. Se concibe que si se estimula el sector con menores tributos se propende por mayor expansión económica, que redunda en mayor recaudo para el Estado, con lo que se puede promover el desarrollo económico y social. No por tener mayor carga tributaria se genera mayor recaudo fiscal.

La experiencia de las grandes reformas económicas en Estados Unidos e Inglaterra en la década de los ochenta, basada en menor carga tributaria y otras reformas estructurales (laborales, financieras, etc.), oxigenaron a la actividad emprendedora. El resultado permitió no sólo sacar a estos países de la fuerte crisis que vivían, sino que les permitió gran direccionamiento y prosperidad de largo plazo.

\section{REFERENCIAS}

BANCO INTERNACIONAL DE RECONSTRUCCIÓN Y FOMENTO (BID). THE BASIS OF A DEVELOPMENT PROGRAM FOR COLOMBIA. John Hopkins University Press, Baltimore, 1952.

CONSEJO DE ESTADO. SALA IV. "SENTENCIA DEL 18 DE MARZO DE 2010". Consejera ponente MARTHA TERESA BRISEÑO DE VALENCIA. Radicado No.: 2500023-27-000-2004-00848-01(16971).

Currie, Lauchlin (1974). DESARROLLO ECONÓMICO ACELERADO. Fondo de Cultura Económica, México.

Froomkin, Joseph (1968). "La administración fiscal de las municipalidades y el desenvolvimiento económico" en imposición fiscal en los países en desarrollo de Richard Bird y Oliver Oldman. Uteha, México.

Hey, Sally (1974) "Ingresos municipales y departamentales en Colombia" en propuesta de una reforma fiscal para Colombia de Musgrave y Gillis. Banco de la República, Bogotá. Tomo III. Pp. 261 y 262. 
PROCESOS URBANOS - Revista de Divulgación Científica Vol. 1 Enero - Diciembre 2014 (103 - 112)

Hicks J. R. y Hicks, U (1968). "Imposición del valor baldío de la tierra" en imposición fiscal en los países en desarrollo de R. Bird y O. Oldman. Uteha, México.

Hirshman, Albert (1968). "El impuesto sobre tierras y reforma agraria en Colombia" en imposición fiscal en los países en desarrollo de Richard, Bird y Oliver Oldman. Uteha, México.

Lewis, Jeffrey D. (1979). Consolidated public finance in Bogotá and Cali, Colombia. Banco Mundial y Corporación Centro Regional de población, Bogotá. mimeógrafo.

Linn, Johannes (1979). "The incidence of urban property taxation in Colombia" en the taxation of urban property in less developed contries de Roy W. Bahl. University of Wisconsin, Madison.

Llorente Martínez, Rodrigo (1972). "Exposición de motivos al proyecto de ley por medio del cual se propuso sobretasa sobre el impuesto predial para asegurar financiación de la educación primaria". Anales el Congreso, Bogotá.

Low Murtra, Enrique y Gómez Ricardo, Jorge. (1986). Política Fiscal. Universidad Externado de Colombia, Bogotá. Cap. V.

MINISTERIO DE HACIENDA Y CRÉDITO PÚBLICO. Estudio soporte al proyecto de ley por medio del cual se propuso crear sobretasa al impuesto predial para financiar de la educación primaria a todo niño con edad escolar". Mimeógrafo. No. (b)-6025hf47-1972. Biblioteca oficina de planeación y estudios económicos y fiscales, Bogotá, 1972.
Netzer, Dick ( 1966). "Some aspects of local government finance in Colombia" en economic development series report. No. 151. Harvard University, Cambridge, 1966.

ORGANIZACIÓN DE ESTADOS AMERICANOS (OEA) Y BANCO INTERAMERICANO DE DESARROLLO (BID). "La política tributaria en cuanto determinante de la productividad de la tierra" en reforma tributaria para América Latina. Documento y conclusiones de la III. Conferencia Interamericana sobre Tributación, México 1972. OEA, Washington, 1973. p. 239.

Pareto, Vilfrido (1946). Manual de Economía Política. Atalaya, Buenos Aires.

Simons, Henry (1936). Personal income taxation. University of Chicago, Chicago. Cap. I.

Urrutia, Miguel ( 1974). "La distribución del ingreso en Colombia" en lecturas sobre desarrollo económico colombiano de Fedesarrollo. Fedesarrollo, Bogotá. p.17.

Vásquez Jaime (1971). "El avalúo catastral y la aplicación del impuesto a la renta al sector agropecuario" en V Seminario Técnico del Centro Interamericano de Administradores Tributarios. CIAT, Bogotá. 